\title{
The experience property frame work: a misleading paradigm
}

\author{
Martine Nida-Rümelin ${ }^{1}$
}

Received: 18 August 2015 / Accepted: 9 May 2016 / Published online: 1 July 2016

(C) The Author(s) 2016. This article is published with open access at Springerlink.com

\begin{abstract}
According to the experience property framework qualia are properties of experiences the subject undergoing the experience is aware of. A phenomenological argument against this framework is developed and a few mistakes invited by the framework (concerning our understanding of consciousness, self-consciousness and the relation of the two) are described. An alternative to the framework, the framework of experiential properties (where experiential properties are properties of the experiencing subject) is presented and defended as preferable. It is argued that the choice between these two frameworks makes a substantial difference for theoretical purposes.
\end{abstract}

Keywords Qualia · Phenomenal consciousness · Self-consciousness · Experience property framework $\cdot$ Experiential properties $\cdot$ Dualism

\section{Introduction}

The fact that there is something that it is like for the experiencing subject to undergo an experience is commonly expressed by saying that experiences have qualitative character. This is usually taken to imply that experiences have properties, so-called qualia, which constitute what it is like to undergo the experience at issue.

This common framework, the experience property framework, will be challenged in the present paper. It is a mistake, or so I will argue, to approach philosophical issues about consciousness within this established paradigm. Experiences have 'qualitative character' only in the sense that they involve an experiencing subject who instantiates

$\triangle \quad$ Martine Nida-Rümelin

martine.nida-ruemelin@unifr.ch

1 Département de philosophie, Université de Fribourg, Avenue de l'Europe 24,

1700 Fribourg, Switzerland 
experiential properties - properties such that there is something that it is like to have them. Philosophical issues about consciousness should therefore be approached by focussing on experiential properties and the status of those who can have them: experiencing subjects. This framework, the framework of experiential properties, should be chosen in order to avoid mistakes that are invited by the experience property framework, or so I will argue in the present paper.

Most philosophers involved in this debate apparently tend to think that it does not matter which of the two frameworks just mentioned one chooses for theorizing about consciousness. But that choice, as will be argued in what follows, makes a substantial difference. There is good reason to abandon the experience property framework in favour the framework of experiential properties, or so I will argue. ${ }^{1}$

\section{Experiences and experiential properties}

Experiences are a subclass of events. Events may be understood as involving things which instantiate properties. Subclasses of events can be distinguished by the kind of individuals involved in the event and by the kind of properties they instantiate. The subclass of experiences can be characterized by saying that the individuals involved are experiencing subjects who instantiate experiential properties. For instance, your experience of blue in a given moment consists of you (an experiencing subject) instantiating the experiential property of being phenomenally presented with blue. An analogous account can be given for every experience.

In order to explain what distinguishes experiences from other events, one thus must say what distinguishes experiential properties from other properties and experiencing subjects from other individuals. Here I will focus on experiential properties. In a first approximation, one may say that experiential properties are characterized by the fact that there is something that it is like to instantiate them. But this characterization covers too much. There is something that it is like to live in Switzerland, since living in Switzerland is typically associated with visual experiences of particular landscapes, with feeling safe in daily life, etc. But living in Switzerland is not an experiential property in the intuitive sense at issue; it does not by itself characterize what it is like to have that property; it determines only indirectly what it is like for a subject who has that property to live its present life. To exclude examples of this kind one must say that to have an experiential property partially constitutes what it

\footnotetext{
1 The experience property framework is standardly used in the ongoing debate about consciousness. Accordingly, qualia are introduced as qualitative properties of experiences or states (the difference does not matter for the arguments of this paper) in various encyclopedia entrances, see for instance Byrne (2004/2015), Kind (2008) and Tye (2015) and in many influential articles (e.g. Jackson 1982; Chalmers 2002). They are rarely introduced as properties of subjects (see for such an exception Carpintero 2003). It is sometimes noted in passing that nothing of relevance for the author's purposes depends on that choice (see for instance Chalmers 2002, footnote 3). I am not aware of any explicit discussion in the literature of whether or not anything substantial depends on the choice between qualia as properties of experiences (or states) and qualia as properties of experiencers.
} 
is like for the subject who has the property at a given moment to live through that moment. $^{2}$

Most philosophers agree that experiences can be categorized into different phenomenal kinds according to their phenomenal character. But how do we understand what is constitutive for membership of an experience in a particular phenomenal kind as opposed to another? The answer should be obvious. We understand what makes it the case that an experience belongs to a specific phenomenal kind by understanding which experiential property the subject involved instantiates and by understanding what having that property amounts to. For instance, we understand what it is for an experience to be a red experience on the basis of our understanding of what it is for a subject to be phenomenally presented with red. Any phenomenal categorization of experiences is based in this way conceptually and metaphysically on experiential properties. Furthermore, to say that an experience has qualitative character at all is to say that it is an event which involves the instantiation of some experiential property or other. The notion of qualitative character is thus only understandable on the basis of some prior understanding of what it is for a property to be experiential.

It follows that reflection about consciousness unavoidably involves reflection on experiential properties. But experiential properties do not explicitly occur within the experience property framework; they play their conceptual role only under the surface in the way just described. Based on this observation one may formulate an argument from conceptual and metaphysical priority against the experience property framework which relies on a plausible general principle: if a given theoretical framework (a) uses more fundamental concepts than another and if (b) the phenomena referred to by the concepts of the former are more fundamental than the phenomena referred to by the latter, then —other relevant aspects such as terminological simplicity being equal-it is advisable to prefer the former framework.

The argument from conceptual and metaphysical priority will, however, convince only few of those who are used since decades to the experience property framework. Many of them will think that nothing substantial depends on the choice between the two frameworks. Thinking so commits one to the claim that the following conditions are all satisfied:

(1) Any claim which has intuitive appeal when formulated in one of the two frameworks is as attractive, intuitively, when formulated in the other framework.

(2) Rational evaluation of an argument when formulated in one of the two frameworks leads to the same result as rational evaluation of the corresponding argument when formulated within the other.

(3) Any claim that can be formulated in one of the two frameworks has a corresponding formulation within the other framework.

(4) The two frameworks are equally adequate to the phenomena they conceptualize.

\footnotetext{
2 Philosophers used to the experience property framework may well accept this explication of what it is to have an experiential property and yet add that having an experiential property is to be in a state or to have an experience with qualia and conclude that the framework of experiential properties collapses into the experience property framework after all. A detailed argument for the claim that no such explication of experiential properties is available is developed in this paper (Sects. 3-12).
} 
If all these conditions were satisfied, then the two frameworks could be considered to be equally acceptable dialects of one and the same theoretical language. The argument from conceptual and metaphysical priority would then have little weight. There would be no strong reason to abandon the experience property framework. To the contrary, the fact that it has been used since decades would then be reason for going on conceptualizing one's thoughts within that framework and for communicating among philosophers using its well-established terminology. However, the assumptions (1) to (4) formulated above are in fact unfounded-as will be plain, I hope, by the end of this paper.

\section{A fallacious argument in favour of the experience property framework}

Many find it natural to think within the experience property framework. This is in part due to habit and also to the fact that common sentences used in natural language (e.g. "this was a horrible experience") appear to support it. But there is perhaps a systematic explanation as well: a simple reasoning which may play a role in the background of some people's mind leads from uncontroversial assumptions directly into the experience property framework. One may reconstruct it as follows:

(Premise P1) For an experience to have qualitative character means that there is something that it is like for the experiencing subject concerned to undergo the experience.

(P1) motivates a further premise which is (like P1) a partial explanation of the technical term "qualitative character":

(P2) The qualitative character of an experience constitutes what it is like for the subject concerned to undergo that experience.

To arrive at the experience property framework, a further premise about 'qualitative character' is required:

(P3) For an experience to have qualitative character is for it to instantiate qualitative properties.

(P2) and (P3) imply:

(C) Certain qualitative properties of experiences constitute what it is like to undergo a given experience.

To accept $\mathrm{C}$ is to adopt the experience property framework. If it is right, as will be argued, that there are good reasons to abandon that paradigm, then it is important to become clearly aware of where the above argument goes wrong.

P1 should be read as a stipulation introducing the technical term "having qualitative character". We may assume that there is something that it is like for the subject concerned to undergo an experience just in case that there is something that it is like for the subject concerned to instantiate the properties the instantiation of which constitutes the experience in question. It follows_-presupposing the above explication of 
experiential properties - that P1 says nothing more than this: An experience has qualitative character just in case it consists in the instantiation of experiential properties by a given subject. This is trivially true of all experiences (according to the explication sketched), but this is just as it should be.

While P1 explicates the locution "to have qualitative character" P2 assumes that it makes sense to talk of different qualitative characters for different experiences. This may already appear to come close to attributing genuine qualitative properties to experiences as such. But, in order to stick as close as possible to what we undoubtedly understand well enough, P2 can and should be reinterpreted in a way which reduces, like P1, to talk about experiential properties. According to P2, the specific qualitative character of an experience constitutes what it is like for the experiencing subject to undergo the experience. The 'what it is like for the subject to undergo the experience' is, as we know, constituted by the experiential properties instantiated by the subject involved. It follows that the specific qualitative character of an experience is nothing over and above the experiential properties instantiated by the subject concerned. Made explicit in this way, P2 introduces the term "the qualitative character of an experience" as short for those experiential properties the subject concerned instantiates in the relevant event. There is no other acceptable interpretation of P2 available here, or so I claim, since the 'what it is like locution' in order to be clearly understandable at all must be ultimately understood on the basis of how it is for the subject concerned to have the properties the instantiation of which constitute the experience at issue. So far we have not made any step towards the experience property framework. But we 'jump into' that framework by introducing premise P3.

Premise 3 states that for an experience to have qualitative character is for it to instantiate qualitative properties. Given that 'qualitative character' has already been introduced, by stipulation, through the premises $\mathrm{P} 1$ and $\mathrm{P} 2, \mathrm{P} 3$ requires an argument based on the former premises. But there is no such argument in sight. According to P1, for an experience to have qualitative character is for the experience to be an event which is constituted by the instantiation of some experiential properties by some subject. This 'property' of an event is not in any clear sense a qualitative property of the experience. It is not a genuine 'property' of the experience at all; it need not be mentioned in order to completely describe the relevant event. It suffices to say that the properties instantiated by the subject involved in the event are of a certain type. According to P2 to have specific qualitative character is, for an experience, to involve a subject who instantiates specific experiential properties. Therefore, to say of an experience that it has a specific qualitative character is not to attribute a genuine property to the event which is the experience; it is just a short way to say which experiential properties are instantiated by the subject concerned. Therefore, neither P1 nor P2 can justify $\mathrm{P} 3$. The impression that $\mathrm{P} 1$ and $\mathrm{P} 2$ motivate $\mathrm{P} 3$ turns out to be a cognitive illusion. $\mathrm{P} 3$ is not even compatible with $\mathrm{P} 1$ and $\mathrm{P} 2$ given the way in which the content of P1 and $\mathrm{P} 2$ reduces to claims about experiential properties. The implicit argument people may have in mind in favour of the experience property framework thus turns out to be invalid. But to motivate the claim that the framework should be abandoned we need a positive argument against it. To develop such an argument is the purpose of the rest of this paper. 


\section{The structure of a phenomenological argument against the experience property framework}

The aim of the phenomenological argument here to develop is to show that experiences do not instantiate qualia in the technical sense of the term which is prevalent in the debate about phenomenal consciousness and can be captured by the following claims:

Q1: Qualia are qualitative properties of experiences.

Q2: The qualia of an experience constitute what it is like for the experiencing subject to undergo that experience.

Q3: While undergoing an experience the subject is in some primitive way immediately aware of the qualia instantiated by the experience.

The aim of the argument which will be presented in what follows is not directed against qualia in a different sense which is also present in the debate and often not clearly distinguished from qualia in the technical sense just explained. Examples of qualia in this other sense are colours, sounds, tastes or odours. This is a different use of the term. These items are not properties of experiences, rather they belong to what is present to the subject who undergoes the experience; they belong to its content. For qualia in this sense the term "c-qualia" will be used in what follows. The argument developed in this paper is not directed against the existence of c-qualia. Nor is the argument directed against realism about phenomenal consciousness. I will use the term "qualia" in what follows exclusively for the technical notion captured by the claims Q1-Q3. The aim of the argument is to show that there are no qualia in that specific sense.

Q1 and Q2 are standard formulations when the technical term of qualia is introduced. ${ }^{3}$ Q3, however, will be doubted by some philosophers moved by so-called transparency of experience (see Sect. 18). And Q3 will be doubted with good reason by those who limit the term awareness to something cognitively more demanding, to something which requires some kind of reflection or conceptualisation. However, or so one may argue, the idea that qualia are in some direct, immediate way (which does not require reflection or conceptualisation) present to the subject undergoing the experience, is built into the very idea of qualia. Qualia are supposed to be in some sense 'felt' or 'immediately there for the experiencer' since they 'directly' determine how it is to have the experience. The argument developed below is effective only against those views which try to incorporate this intuition. But, I believe, most philosophers who take the claim that experiences have qualia seriously and believe that it expresses a puzzling fact which leads - at least prima facie — to trouble for physicalism do agree with the intuitive idea expressed by Q3. Arguably, to abandon Q3 would be to lose the intuitive motivation for introducing the technical term 'qualia' in the first place.

\footnotetext{
3 Qualia are introduced as qualitative properties of experiences or states (the difference does not matter for the arguments of this paper) in encyclopedia entrances such as Byrne (2004/2015), Kind (2008) and Tye (2015) and in many influential articles (e.g. Jackson 1982; Chalmers 2002). They are rarely introduced as properties of subjects (see for such an exception Carpintero 2003). It is sometimes noted in passing that nothing of relevance for the author's purposes depends on that choice (see for instance Chalmers 2002, footnote 3). I am not aware of any explicit discussion in the literature of whether or not anything substantial depends on the choice between qualia as properties of experiences (or states) and qualia as properties of experiencers.
} 
To argue for the claim that we are not immediately aware in the relevant intuitive sense at issue in Q3 of any properties fulfilling Q1 and Q2 I will proceed as follows. I will distinguish three familiar ways in which one can be directly aware of the instantiation of a property. I will then argue that in none of those ways we are ever aware of properties fulfilling Q1 and Q2. Since no other ways of being immediately aware of the instantiation of properties are in sight, one may conclude that no properties are ever instantiated which fulfil Q1-Q3 simultaneously.

To answer the phenomenological argument just sketched and developed below the opponent would have to show either (a) that - contrary to the arguments below-we are aware of properties fulfilling Q1 and Q2 in one of the three ways at issue or (b) that some further type of immediate awareness exists, that we are aware of qualia in that further way and that the present phenomenological argument does not generalize to that further type of immediate awareness. I doubt that any of these two strategies can be convincingly carried out.

In preparation of the argument three candidates for immediate awareness of socalled qualia will now be briefly presented in the following three short sections.

\section{Immediate awareness in the sense of phenomenal presence}

The clearest cases of phenomenal presence are episodes in which a subject is presented with a c-quale like, for instance, a particular slightly reddish blue, the sound of a violin, the smell of basil or the taste of ginger. It is not always the case that in being phenomenally presented with a c-quale there is a corresponding property actually instantiated in one's environment. The sound of a violin as well as tastes and sounds can be hallucinated. Nor is it necessarily the case that some property appears to be instantiated in one's environment when one is phenomenally presented with a c-quale. For instance, a person can be phenomenally presented with red with her eyes closed without thereby being under the impression that there is something around which actually is red. The ontological status of what is phenomenally present raises puzzling questions which however need not be addressed here. No answer to these questions will be required for the argument to be developed. ${ }^{4}$

\section{Immediate awareness in the sense of perceptual awareness}

In perception we are aware of the properties of things around us in a way which intuitively deserves to be called immediate. We seem to have direct access to certain properties of things just by looking. The intuition of immediacy or directness here at issue can be cashed out in various ways and there is no agreement among philosophers about how it should be accounted for. Perhaps an important element concerns the way we are related to the properties of things along the following lines: with respect to certain properties things surrounding us cannot change without that change showing up in how they appear to us. A certain sense of immediacy of perception can thus perhaps

\footnotetext{
4 A specific view about the ontological status of phenomenally presented 'objects' is proposed in my paper (2011).
} 
be captured by saying that there is an appropriate counterfactual dependence between how things are and how things appear when they are perceptually accessible to us. ${ }^{5}$ But these facts about our actual perceptual access to the properties of things cannot be all there is to be said about the immediacy at issue. Perception also involves a certain phenomenology of directness. We appear to have such direct access and so the question arises what exactly appears to be the case when it seems to us that we have direct access through perception to properties of real things in our environment. Perhaps we are experientially aware of the counterfactual dependence mentioned before. Or perhaps an adequate description of that particular perceptual phenomenology of directness requires mentioning the apparent absence of inference or the apparent absence of reason for doubt. No theoretical account of our so-called perceptual openness to the world—as these intuitions about perceptual directness are sometimes expressed-will be needed, however, for the argument to be developed below. ${ }^{6}$

\section{Immediate awareness in the sense of primitive awareness}

Many philosophers argue that there is a sense in which experiences are necessarily conscious. ${ }^{7}$ I agree with this claim. The point can be put as follows: experiences are such that, undergoing the experience necessarily involves that the subject is aware of undergoing it. Let us call this kind of awareness primitive awareness. By stipulation, primitive awareness refers to the kind of awareness for which the claim is true that, in that particular sense of awareness, it is impossible to undergo an experience without being aware of undergoing it.

Primitive awareness, understood in this way, is not the result of so-called introspection or of phenomenal reflection (as I prefer to say for reasons that will become clear below). To undergo an experience does not necessarily involve reflecting upon it and so primitive awareness does not involve phenomenal reflection. Since primitive awareness is arguably required for phenomenal reflection as a precondition primitive awareness may be called pre-reflective. Primitive awareness does not require conceptualizing the experience one undergoes but it is plausibly required for forming a concept of a given experience on the basis of one's own experience. Therefore it is apt to call primitive awareness pre-conceptual.

\section{An account of primitive awareness}

Primitive awareness is thus a pre-reflective and pre-conceptual awareness of one's own experience such that it is impossible to undergo an experience without being primitively aware of undergoing it. Two possibilities are left open when one accepts

\footnotetext{
5 This proposal is developed and defended in my paper (2011).

6 The intuition of 'perceptual openness' to the world and attempts at accounting for it is presented in detail by Tim Crane in his encyclopedia entrance (2005/2011); for an epistemological account of this intuition compare Soldati (2013).

7 A representationalist version of the thesis is famously defended by Brentano (1874) (compare the exposition of Brentano's view in Soldati, forthcoming) and adopted in different versions by contemporary authors (representationalist versions are developed e.g. in Levine 2006; Horgan and Kriegel 2007; Kriegel 2009). A non-representationalist account is defended in various publications by Dan Zahavi, see e.g. his book (2014).
} 
the claim that experiencing and being primitively aware of experiencing cannot be separated: (1) Primitive awareness of experiencing is something over and above experiencing itself; these two different 'aspects' of a subject's conscious life are however correlated with necessity. (2) Primitive awareness of experiencing is nothing over and above experiencing. Experiencing $i$ s already being primitively aware of experiencing. Primitive awareness of experiencing is thus neither a part of experiencing nor anything in addition to experiencing.

I here assume the second option. ${ }^{8}$ If the second option is correct, then there is a way to say more simply what a subject is aware of when it is 'aware of undergoing a particular kind of experience': A subject is aware of undergoing a particular kind of experience when it is aware of having the corresponding experiential property. For instance, to be aware of undergoing a red experience is to be aware of being phenomenally presented with red. In general, when an experience consists in a subject's instantiation of experiential properties $\mathrm{E}$, then a subject's primitive awareness of the experience is nothing more or else than the subject's primitive awareness of instantiating the experiential property E. Choosing the above second option (being aware of experiencing is nothing over and above experiencing) we thus arrive at a further way to characterize experiential properties. Experiential properties are those properties which cannot be instantiated by any individual without that individual being pre-conceptually and pre-reflectively aware of instantiating them. In other words: to have an experiential property is to be aware of having it.

This result may appear paradoxical. One is tempted to think that no property $\mathrm{P}$ can be such that having $\mathrm{P}$ and being aware of having $\mathrm{P}$ is one and the same. But here it helps to remember what experiential properties are: experiential properties are such that, by their nature, the instantiation of such a property by a subject at a given moment $\mathrm{m}$ partially constitutes what it is like for the subject to live through moment $\mathrm{m}$. Now there is an obvious and clear sense in which any subject is aware of what it is like for it to live through moment $\mathrm{m}$ just by living through that moment. One may say that the subject is immediately aware-without reflection and without conceptualization - of every specific aspect of what it is like for it to live through moment $\mathrm{m}$ while it is living though moment $\mathrm{m}$. To admit this is already to admit what needs to be seen: given the nature of experiential properties, to be aware of such an aspect is to be aware of having the corresponding experiential property. This is why having an experiential property just is being aware of having it.

The account of primitive awareness sketched in the present section will not be presupposed in the phenomenological argument here to develop. Since the account uses the framework of experiential properties it would arguably be question begging to base an argument against the experience property framework on this particular understanding of primitive awareness. This section serves to give some idea to the reader of how the alternative to the experience property framework can be used for theoretical purposes. The section will prove useful at the end of this paper (see Sect. 20 entitled "Concluding remark").

\footnotetext{
8 Arguments for this option are developed in my papers (2014), (2016) and in Chap. 8 of my book (in preparation).
} 


\section{Candidates for properties fulfilling Q1 and Q2}

According to the experience property framework there are qualitative properties of experiences (constraint Q1) which are constitutive of what it is like to undergo the experience instantiating them (constraint Q2) and of which we are immediately aware in undergoing the experience which has those properties (constraint Q3). To prepare the argument against that claim let us see what properties of experiences can be said to fulfill the first two constraints. To complete the argument it must then be shown that these properties do not fulfill the third constraint (we are in not immediately aware of them in any of the three senses explained while undergoing an experience instantiating those properties).

It will suffice to search for properties fulfilling Q1 and Q2 for the example of red experiences; it will be obvious how the result generalizes to other cases. So we are searching for a qualitative property of red experiences which is constitutive of what it is like to undergo a red experience. Put in this way it is not yet obvious how one must proceed to find the answer. At this point it helps to remember that red experiences form — as is quite generally agreed - a phenomenal kind. This assumption can be restated as follows: what it is like to undergo a red experience is constitutive for membership in the class of red experiences. It follows with constraint Q2 that the property we are searching for coincides with the property which is constitutive for membership in the class of red experiences. In order to find a property fulfilling Q1 and Q2 we must therefore find the property of events which is constitutive of membership in the class of red experiences. And this latter property is easy to find. An event falls in the class of red experiences in virtue of the fact that it consists in some experiencing subject being phenomenally presented with red. This is then the property we are searching for: being an event such that the event consists in someone's instantiation of the relevant experiential property (in the case of red experiences: of being phenomenally presented with red). Given the premises just used, this property is the only property which fulfills Q2 and therefore the only candidate for 'a quale' common to red experiences in the technical sense here at issue. To simplify exposition this property of red experiences will be called $R_{E}$ in what follows.

The argument could be finished quickly by saying that $R_{E}$ is not a qualitative property and so does not fulfil Q1. But this argument would not be strong enough. The opponent might be ready to abandon the claim that qualia are qualitative properties or start a sophisticated debate about what it is for a property to be qualitative. A more important and revealing objection against the experience property framework focusses on $\mathrm{Q} 3$, the constraint of immediate awareness.

\section{$10 R_{E}$ is not phenomenally presented}

If red experiences have some quale (in the technical sense at issue) in common, then that 'quale' is the property $\mathrm{R}_{\mathrm{E}}$. This is the result of the preceding section. It follows that every subject undergoing a red experience would have to be phenomenally presented with $R_{E}$ if our awareness of the qualia of our experiences were awareness in the sense of phenomenal presence. 
So we must ask: is $\mathrm{R}_{\mathrm{E}}$ phenomenally present to every subject who is phenomenally presented with red? The answer is quite obviously negative. It is phenomenologically obvious, or so I would like to claim, that no instantiation of the complex property $\mathrm{R}_{\mathrm{E}}$ is ever phenomenally present to us and it is even more obvious that such presence is not necessarily correlated to undergoing a red experience as it would have to be if the claim at issue were correct. The argument to develop for the claim that phenomenal presence is not an acceptable candidate for awareness of supposed qualia could simply end here. But perhaps it is more helpful to dig a little deeper and to ask: why is it so obvious that we are not phenomenally presented with $\mathrm{R}_{\mathrm{E}}$ in every red experience? In other words: what makes the contrary thesis so obviously inadequate?

A plausible idea is to say that $R_{E}$ is not of the right kind for being phenomenally presented. It is a complex property and properties which can be phenomenally presented to someone, so one might say, are simpler. But this does not quite capture the crucial point. Mere complexity is not the problem. We are, for instance, capable of being phenomenally presented with instantiations of three-dimensional shape properties which, when analyzed properly within mathematical terminology, are highly complex too.

A second way to say why the thesis that $R_{E}$ is phenomenally present is phenomenologically inacceptable is to say this: the thesis requires that in every red experience we are not only phenomenally presented with red, but also phenomenally presented with a further property. A little phenomenological reflection, however, reveals, or so one may argue, that there simply is no such further property phenomenally presented to us alongside red in every experience of red. This is, in my view, as far as it goes, a valid phenomenological argument against the thesis to reject.

A third idea which comes to mind is related to the unusual nature of the property $R_{E}$. $\mathrm{R}_{\mathrm{E}}$ is not, as one might express the thought, a genuine feature of experiences. Experiences fall under the concept $\mathrm{R}_{\mathrm{E}}$ only in virtue of the fact that the subject involved instantiates the corresponding experiential property (being presented with red). There is no feature of the experience as such, so to speak, which could be phenomenally presented since what makes the attribution of $\mathrm{R}_{\mathrm{E}}$ to a given experience correct is not the instantiation of such a feature by the relevant event (the experience) but rather the simple fact that the subject involved in the experience is presented with red. It follows, or so one may argue, that being phenomenally presented with $\mathrm{R}_{\mathrm{E}}$ while undergoing a red experience cannot be other than being phenomenally presented with the instantiation of being oneself phenomenally presented with red. Let us be clear about what that would mean. It does not just mean that in being phenomenally presented with red we are also somehow aware of being presented with red which is a claim one should not deny. Rather, it would mean that one's own instantiation of being presented with red would be phenomenally present to the subject which means it would be given to the subject in the way in which redness and other c-qualia can be given in experience. This sounds absurd. But why does it sound absurd?

The reason is, or so I suggest, that awareness of one's own experiential properties is not to be misunderstood as experiencing one's own properties. Let me try to say this more clearly. Every experience has a subject-object structure. In every experience something is given to the experiencing subject even though that something need not actually exist. We may call this feature of experiences basic intentionality. Basic inten- 
tionality does not consist in a relation between the subject and something else, since what is presented might not be real and relations require the existence of the relevant relata. One may say that basic intentionality is the metaphysical structure of every experience. I cannot try to give a more theoretical account of basic intentionality here but I trust it will be clear to the reader on the basis of examples what general feature of experiences the term is meant to refer to. ${ }^{9}$

If our awareness of our own experiential properties were to be understood as experiencing the instantiation of those properties, then that awareness would have to exhibit basic intentionality. The instantiation of one's own experiential property would have to be present as an additional object to the subject concerned. But this is obviously not the case. In having a red experience, red is given 'as an object' in the relevant sense. But one's own instantiation of being presented with red does not occur as a 'further object' in the stream of consciousness. In other words: awareness of one's own experiential properties does not exhibit basic intentionality. ${ }^{10}$

On the basis of these reflection we may perhaps say briefly and more generally what is wrong about the idea that $\mathrm{R}_{\mathrm{E}}$ is phenomenally present to the experiencing subject undergoing a red experience: whatever the awareness of $\mathrm{R}_{\mathrm{E}}$ (in undergoing a red experience) might amount to (if there is such an awareness at all) it certainly is not an awareness exhibiting basic intentionality. The property $R_{E}$ does not show up as an element in the stream of consciousness. But it would have to show up as an element in the stream of consciousness if it was phenomenally present since phenomenal presence does exhibit basic intentionality.

\section{We are not perceptually aware of $R_{E}$}

Perceptions are experiences. They exhibit basic intentionality. This already excludes by the same argument given for the case of phenomenal presence that we are perceptually aware of the property $R_{E}$. If we were perceptually aware of $R_{E}$, then $R_{E}$ would be given to us in red experiences as a further object showing up in the stream of consciousness. But a little phenomenal reflection reveals that this is not so. Therefore, we are not perceptually aware of $R_{E}$.

One might think that the argument sketched above is not required since there is a simpler argument available: we are not perceptually aware of $R_{E}$ because we are only perceptually aware of things revealed by the senses and instantiations of $R_{E}$ by experiences are not revealed by the senses. But to argue in this way would be to oversimplify the issue. It could be, after all, that conscious beings have what has sometimes been called an 'inner sense' which allows them to track properties of inner

\footnotetext{
9 The concept of basic intentionality is introduced in greater detail in my paper (2014) and in Chap. 8 of my book "Conscious Individuals" (in preparation).

10 This insight has been expressed by a number of philosophers in different ways across the history of philosophy. In contemporary philosophy of mind it has been defended among others by Dan Zahavi (see e.g. Zahavi 2014), by Charles Siewert who calls the pre-flective awareness here at issue of our own phenomenal states 'non-objectual' (compare e.g. Siewert 2012) and Brie Gertler (compare e.g. her criticism of Kriegel in Gertler 2012).
} 
events. If this were so then our awareness of qualia would be sufficiently similar to perception for being called perceptual awareness in the present context.

The idea that our awareness of the qualitative properties of our own experiences is something like such an inner tracking is perhaps quite forcefully present in the background of many people's mind. The metaphor of such inner tracking which treats awareness of so-called qualia in analogy to perceptual awareness is a bad metaphor, however, and it leads to a distorted picture of phenomenal consciousness: the metaphor of such 'inner tracking' would be adequate only if awareness of those 'qualia' had the structure of basic intentionality. But as argued before, this is not the case.

\section{Primitive awareness does not help}

The last candidate for immediate awareness of qualia understood as properties of experiences is primitive awareness. To complete the argument it will suffice to show, for the case of red experiences, that we are not primitively aware of $R_{E}$ when undergoing a red experience or rather that we are only aware of $\mathrm{R}_{\mathrm{E}}$ in the sense that we are aware of instantiating the relevant experiential property, being presented with red. Both results are equally good for the present purposes. It does not matter in the context of an argument against the experience property framework whether the only candidate for a quale $\left(\mathrm{R}_{\mathrm{E}}\right.$, in our example) does not fulfill Q3 (we are not immediately aware of it when undergoing a red experience) or whether the only sense in which we are aware of that 'quale' in such an experience can be reduced to awareness of having the relevant experiential property (to awareness of being phenomenally presented with red).

According to the account of primitive awareness proposed in Sect. 8, we are only primitively aware of our own present experiential properties. This assumption is part of the framework of experiential properties which is an alternative to the framework the argument is supposed to undermine. It would therefore be question begging to use this assumption here. In the following argument for the claim that we are not primitively aware of qualia (understood as properties of experiences) no particular assumption about primitive awareness will be used beyond what has been stipulated in order to introduce that notion: primitive awareness is the kind of awareness a subject has necessarily of undergoing an experience while it undergoes the experience.

What remains to be shown in order to complete the argument (for the special case of red experiences) is this: either we are not primitively aware of $R_{E}$ in undergoing a red experience or to be primitively aware of $R_{E}$ is nothing but being aware of being phenomenally presented with red. Given the way primitive awareness has been introduced this amounts to showing the following: either (a) having a red experience does not necessarily involve being aware of $R_{E}$ or (b) having a red experience necessarily involves being aware of $\mathrm{R}_{\mathrm{E}}$ but the latter awareness reduces to being aware of being phenomenally presented with red. In order to argue for this thesis we need to discuss the following claim of necessity $\mathrm{N}$ :

$\mathrm{N}$ : Whenever a subject is phenomenally presented with red, then, necessarily, the subject is aware of an instantiation of the property $R_{E}$.

$\mathrm{N}$ turns into $\mathrm{N}^{\prime}$ when formulated more explicitly: 
$\mathrm{N}^{\prime}$ : Whenever a subject is phenomenally presented with red, then, necessarily, the subject is aware of there being an experience which is such that the subject involved in it is presented with red.

Our aim is to show that $\mathrm{N}$ is either false or its consequence does not tell us anything more than that the subject is aware of being phenomenally presented with red.

One might be tempted to reject $\mathrm{N}^{\prime}$ arguing that the description given of what the subject is necessarily aware of is too complicated. I tend to think that this is an adequate reaction. But let us assume, for the sake of argument, that $\mathrm{N}^{\prime}$ cannot be rejected for that simple reason. Then the best strategy to reach the desired result is to show that, for $\mathrm{N}^{\prime}$ to be acceptable, the awareness at issue of an instantiation of property $\mathrm{R}_{\mathrm{E}}$ must be understood as being nothing over and above awareness of one's own experiential property of being presented with red. This can be done in two steps: (a) First one argues that for $\mathrm{N}^{\prime}$ to be acceptable at all it must be interpreted as presupposing the claim that every subject phenomenally presented with red is thereby aware of being phenomenally presented with red. (b) Second one argues (on that basis) that $\mathrm{N}^{\prime}$ must be interpreted as not attributing any further awareness to the subject beyond the subject's awareness of its own experiential property.

To take the first step of the argument (step (a) above), it is helpful to ask what reason one might possibly have for accepting $\mathrm{N}^{\prime}$, that is for saying that any subject phenomenally presented with red thereby necessarily is also aware of the occurrence of some experience which involves a subject who is phenomenally presented with red. Quite obviously the only plausible motivation for this claim is to say that a subject who is phenomenally presented with red is thereby aware of the occurrence of an experience with the relevant 'property' because it is aware of being itself phenomenally presented with red. Obviously, the subject can have no other basis for being aware of the occurrence of an experience which involves a subject who is presented with red, than its awareness of being involved in such an experience, and that latter awareness $i s$ awareness of being phenomenally presented with red. It follows that $\mathrm{N}^{\prime}$ is only acceptable if one reads it as presupposing that a subject who is phenomenally presented with red thereby is also necessarily aware of being phenomenally presented with red. This completes the first step of the argument.

In order to see why $\mathrm{N}^{\prime}$ can only be accepted if awareness of $\mathrm{R}_{\mathrm{E}}$ is taken to reduce to awareness of instantiating the relevant experiential property let us consider what the opposite thesis would amount to. Given the result of the first step of the argument just presented, to claim that such awareness does not reduce to awareness of being phenomenally presented with red is to say that whenever a subject is phenomenally presented with red then (a) it is aware of being phenomenally presented with red and in addition to this (b) the subject is also aware of some experience occurring which is such that the subject involved in it is phenomenally presented with red. In certain cases it would be adequate to describe a subject's situation by both (a) and (b). Here is such a case: the subject at issue has acquired the general concept of being presented with red. Therefore, whenever the subject is phenomenally presented with red the subject also conceptualizes the experience it is presently undergoing as involving someone who is phenomenally presented with red. But being phenomenally presented with red, or so I presuppose here as uncontroversial, does not require having developed a concept 
of being phenomenally presented with red. Therefore, the interpretation of (b) just sketched is not available to the opponent who wishes to defend $\mathrm{N}^{\prime}$.

The following hypothesis is highly plausible (I leave it to the opponent to prove the opposite): on every interpretation of condition (b) which turns it into a condition claiming more than (a), condition (b) is not necessarily fulfilled by a subject who is phenomenally presented with red. If this is correct, then it follows that the necessity claim here at issue ( $\mathrm{N}$ resp. $\mathrm{N}^{\prime}$ above) is only acceptable if one interprets awareness of $\mathrm{R}_{\mathrm{E}}$ as nothing over and above awareness of being phenomenally presented with red. This completes this part of the argument.

All three candidates for immediate awareness of qualia (phenomenal presence, perceptual awareness, primitive awareness) have now been considered. None of them turned out to be the kind of awareness at issue when we are, as the experience property framework suggests, aware of supposed qualia of experiences. So we could not find properties fulfilling the constraints Q1, Q2 and Q3 simultaneously. It follows that the experience property framework is unacceptable.

\section{Experiences and brain processes}

The argument developed in the preceding sections against the experience property framework presupposes a particular account of what experiences are. It presupposes that experiences are instantiations of experiential properties by experiencing subjects. Perhaps the opponent could reply putting this assumption into doubt by saying something like this: experiences are inner processes. According to all we know today on the basis of science, these inner processes cannot be other than neural processes in the brain. It follows that the qualia of experiences (understood as properties of experiences) are properties of brain processes. The property $R_{E}$ which has been assumed to be the only candidate for a quale in the technical sense at issue is not a property of brain processes. Therefore, the argument does not go through.

Various things should be said in response to this counterargument. First, the reply suggests that experiences are "inner" in a literal sense of happening "inside the limits of our body". It is only on this assumption that science makes it highly plausible that they cannot be other than neural processes happening in the brain. Now there might be theoretical arguments for the view, as they have been developed by so-called identity theorists, that experiences are brain processes and therefore something going on within the limits of our body or brain. But putting those materialist arguments aside, the assumption that experiences are inner events in the literal sense at issue does not have any independent motivation. It seems to be quite common to think, however, that there is phenomenological motivation for the claim that experiences literally happen inside. This is probably due to the widespread thought that attending to what it is like to undergo one's present experience amounts to attending to some process which appears to happen inside. The term "introspection" invites this idea. Reflection on concrete examples reveals, however, that this is a mistake. We can attend to what it is like to see a specific color on a given surface. We then attend to the surface and its apparent color and thereby to what it is like to see that color. We are not then under the impression of attending to some process which literally happens inside our 
brain or body. No such inner ongoing is phenomenally present in the first place as something which happens inside. Directing one's attention onto some such process which appears to happen inside would however require that such an apparently inner process is phenomenally present. One is perhaps easily misled by theoretical prejudice into the assumption that phenomenal reflection involves an 'apparent look towards inside' in the literal sense of 'inside'. But a further factor may play a role: many test their intuitions about experiences and our awareness of them using the example of pain. And attending to how it is like to undergo one's pain experience does, in typical cases, involve attending to a process which seems to happen inside. For instance, when while suffering from a head ache, a person attends to the qualitative character of her experience, then she attends to a process which is apparently located inside his or her head. But we need to carefully distinguish in such a case between the process which apparently happens in the head (the object of the experience) and the experience itself (the subject's 'experiential awareness' of that process, the event consisting in the phenomenal presence of that object to the subject). With this distinction in mind, the idea that pain experience can be used to phenomenologically motivate the claim that experiences happen inside reveals itself as based on confusion.

Second, one may wonder what a proponent of the counterargument presently under consideration has to say about the thesis that experiences are instantiations of experiential properties by experiencing subjects. Brain processes are instantiations of patterns of neurological activities in groups of neurons. It will be quite uncontroversial that such groups of neurons are not to be identified with experiencing subjects. So the identification of instantiations of experiential properties by experiencing subjects with brain processes is untenable. There are two ways in which the opponent might respond. A first strategy is to deny that experiences are instantiations of experiential properties by experiencing subjects. The second is to agree that experiences are instantiations of experiential properties and to refine the thesis that experiences are also brain processes along the following lines: experiences are those events which consist in the instantiation of the property of the whole brain (or the whole body) to realize a particular pattern of neural activation in a particular region. The first strategy is not convincing, or so I would argue, since we understand what we wish to refer to using the term "experiences" only by considering concrete examples and these examples are all of the following kind: some subject instantiates some specific experiential property. The second strategy is therefore the one the opponent should choose. What follows for the counterargument under consideration?

An immediate consequence is that experiences are not (on that proposal) brain processes after all since brain processes are realizations of patterns of neural activation in regions of the brain. The counterargument must be refined accordingly. But this does not change much or so it seems at first sight. The opponent must reformulate the objection by saying something like this: experiences are instantiations of neural properties of brains as a whole (or of biological organisms as a whole). The property $\mathrm{R}_{\mathrm{E}}$ is not a property of such instantiations of neural properties by brains or organisms. Therefore the argument does not go through.

But now a counter-objection to the counterargument at issue is readily available. Since the opponent here under consideration accepts that experiences are also instantiations of experiential properties by experiencing subjects the argument in its original 
form is not undermined at all. The property $R_{E}$ has been shown to be the only candidate for a quale and the argument for this view was based on the assumption just mentioned about the nature of experiences which the opponent here under consideration is ready to concede. So the counterargument at issue collapses.

\section{Supposed qualia of brain processes}

The idea that experiences are brain processes is so common in the present debate that the issue about phenomenal consciousness is often understood as concerning the question about whether or not brain processes have qualia (the eliminativist denies this) or about whether or not those special properties of brain processes are physical or nonphysical in their metaphysical nature. A background assumption shared by many (not only by materialists but also by dualists and philosophers who take the latter option seriously) is this: experiences are inner events in the literal sense. Therefore it is quite unproblematic to identify experiences with neural processes happening in the brain. In the preceding section arguments have been given for the claim that-contrary to what is often assumed-there is no phenomenological motivation available for the view that experiences are spatially located within the limits of our bodies and a reply to a counter-argument based on the identification of experiences with brain processes has been given. It is therefore not necessary for the argument her presented against the experience property framework to consider the option that qualia are properties of brain processes. What has been said in Sect. 13 would suffice to justify putting this option aside.

However, the contrary assumption (qualia, if they exist, are properties of brain processes) is so widespread that it would not be wise to proceed in this manner. So let us assume, for the sake of argument, that a version of the experience property framework must be taken into account which combines the claims Q1-Q3 (qualia are qualitative properties of experiences constituting what it is like to undergo an experience and of which we are immediately aware when undergoing the relevant experience) with the additional claim that experiences are brain processes.

The reformulation of the argument as directed against this version of the experience property framework will not take much space here, however, since all steps of the original argument have an obvious and equally convincing counterpart.

The first step in the reformulation at issue is to find the property corresponding to $\mathrm{R}_{\mathrm{E}}$ in the original argument. To simplify exposition let us introduce a term for the class of brain processes at issue and let us call them R-processes. It is agreed that R-processes form a phenomenal class: for a brain process to belong to that class is for it to be such that there is a specific way it is like to have a brain in which that process takes place. That 'specific way it is like' is captured by saying that the subject concerned is phenomenally presented with red. The essential commonality of R-processes can thus be formulated like this: it is the property of a brain process consisting in the fact that the subject concerned is phenomenally presented with red. "The subject concerned" is used here to refer to the subject who has the brain in which the process takes place. So now we have an account of the property $R_{E^{\prime}}$ which is constitutive for membership in the class of R-processes. On the other side we know that the supposed quale typical 
for R-processes is what is assumed to be constitutive for membership in the class of $\mathrm{R}$-processes. It follows that $\mathrm{R}_{\mathrm{E}^{\prime}}$ is the only candidate for the supposed quale common to all R-processes.

The parallel argument to be presented here must continue showing that we are neither phenomenally, nor perceptually, nor primitively aware of $\mathrm{R}_{\mathrm{E}^{\prime}}$ or rather that primitive awareness of $\mathrm{R}_{\mathrm{E}^{\prime}}$, if it exists, reduces to being primitively aware of being phenomenally presented with red. There is however no relevant difference between this argument and the one already presented for the property $\mathrm{R}_{\mathrm{E}}$. The most fundamental objection against the idea that we are phenomenally or perceptually aware of $R_{E^{\prime}}$ is again that both, phenomenal awareness and perceptual awareness exhibit basic intentionality. But again it is clear that the property $\mathrm{R}_{\mathrm{E}^{\prime}}$ does not occur as an element in the stream of consciousness. Therefore awareness of $\mathrm{R}_{\mathrm{E}^{\prime}}$ cannot belong to a kind of awareness which exhibits basic intentionality. It follows that phenomenal awareness as well as perceptual awareness are excluded as candidates for the way in which we are aware of the best candidate for the 'quale' of brain processes, of the property $R_{E^{\prime}}$.

The argument concerning primitive awareness must again be developed in two steps: in a first step one realizes that the only plausible motivation for the claim that being phenomenally presented with red necessarily involves awareness of an instantiation of $\mathrm{R}_{\mathrm{E}^{\prime}}$ relies on the assumption that the person concerned is aware of being phenomenally presented with red. In a second step one argues that no interpretation of "awareness of $R_{E^{\prime}}$ " can be given which satisfies two conditions: (a) on that reading the claim is convincing that being phenomenally presented with red necessarily involves awareness of $\mathrm{R}_{\mathrm{E}^{\prime}}$ and (b) on that interpretation awareness of $\mathrm{R}_{\mathrm{E}^{\prime}}$ is something over and above awareness of being phenomenally presented with red. It follows from the result of the second step that either we are not primitively aware of $\mathrm{R}_{\mathrm{E}^{\prime}}$ when undergoing a red experience or that this awareness is nothing more than primitive awareness of being phenomenally presented with red. This completes the present variant of the argument which leads to the following result: there are no properties of brain processes fulfilling Q1-Q3, so brain processes do not have qualia.

The arguments given so far for the claim that the experience property framework is unacceptable are unlikely to impress anybody used to working within that framework as long as it hasn't been shown why and how the framework leads into serious errors. I therefore briefly describe in what follows a number of mistakes that are invited by that framework. ${ }^{11}$ These mistakes are all frequent and influential in the present debate about consciousness. Sometimes they are quite explicitly expressed, sometimes they influence the way people think 'beneath the surface'.

\section{Confusing different kinds of acquaintance}

All familiar cases of acquaintance are what one might call cases of acquaintance by presentation. Cases of acquaintance by presentation include those and only those examples where something is familiar to a subject on the basis of a mental state or event

\footnotetext{
11 A deeper analysis of how the framework leads to these and other mistakes is presented in Chap. 2 of my book (in preparation).
} 
which exhibits basic intentionality; in that case something is presented to the subject, it occurs as an element in the stream of consciousness the subject is presented with. We are acquainted, for instance, with people by being presented with them through perception, we are acquainted with colours by being presented with them through vision, perhaps we are acquainted with contents of thought by being presented with those contents in theoretical reflection.

It may seem as if acquaintance always requires being presented or having been presented with the item one is acquainted with. But there is an important exception: acquaintance with experiential properties. We are acquainted with experiential properties by instantiating them. For instance, a subject is acquainted with how it is to be phenomenally presented with red by being phenomenally presented with red. But no instantiation of an experiential property is the object here of the subject's experience. No instantiation of 'being phenomenally presented with red' occurs as an item in the stream of consciousness; no such instantiation of being phenomenally presented with red is given to the subject as an object. Nor is there any process or ongoing (e.g. an experience) given as an object to the subject in that case. It is not as if, in addition to red, there also is an experience of red which somehow occurs as an object in the subject's stream of consciousness. The subject is aware of an experience of red when being phenomenally presented with red; the subject is aware of the experience, as we may say, which it is presently undergoing. But that awareness is not to be misunderstood as exhibiting basic intentionality. The subject is acquainted with being phenomenally presented with red by being primitively aware of being phenomenally presented with red. Since primitive awareness does not exhibit basic intentionality, acquaintance through primitive awareness is not a case of acquaintance through presentation. Acquaintance by primitive awareness is thus fundamentally different from usual cases of acquaintance. ${ }^{12}$

Using the experience property framework one risks to be blinded for this fact. The framework portrays acquaintance with different phenomenal kinds of experience as being acquaintance with qualia. Since those qualia are understood as properties of experiences (and thus as properties of some other entity than the subject itself) the picture is forced upon the one who thinks within that framework that qualia are presented to the subject at issue and that therefore the subject is acquainted with qualia by presentation. The framework thus almost unavoidably leads to the fundamental mistake of misinterpreting acquaintance with experiences on the basis of experience as acquaintance by presentation. That mistake leads to further errors. Some of them will be briefly presented in the following sections.

\section{Phenomenal consciousness and self-awareness}

Being aware of what it is like to have an experience by primitive awareness is to be aware of instantiating an experiential property. It is not to be aware of an instantiation of a property by something where that something is given as an object in the expe-

12 Acquaintance with oneself as a subject is likely to be the only other case of acquaintance which is not acquaintance by presentation; compare my paper (2016) and Chap. 10 of my book (in preparation). 
rience or by someone whereby it is left open who that someone is; rather it is to be aware of oneself instantiating the experiential property at issue. Primitive awareness of experiential properties therefore comes along with some form of awareness of oneself, with self-awareness in the specific sense of being aware of oneself.

Awareness of one's experiential properties and self-awareness are thus intimately related or rather, awareness of one's own experiential properties is a form of awareness of oneself. Being phenomenally conscious (which is nothing else than instantiating experiential properties) involves being aware of having those experiential properties (in the sense of primitive awareness) which in turn involves, as just said, being aware of oneself. To be clear about this intimate relation between phenomenal consciousness and self-awareness is crucial for developing an adequate theoretical account of both, phenomenal consciousness and self-awareness. One might say that phenomenal consciousness and pre-reflective self-awareness are just two sides of one and the same 'phenomenon'. That intimate 'relation' becomes clearly visible, as just sketched, within the framework of experiential properties. Contrary to this, the experience property framework hides this intimate 'relation' and makes it difficult to even formulate this important insight.

This disadvantage of the experience property framework is closely related to what has been said in the preceding section about errors concerning acquaintance. According to the experience property framework we are acquainted with phenomenal kinds of experiences by being presented with qualia. Awareness of being phenomenally presented with red is replaced, within that framework, by awareness of a quale common to red experiences. Being presented with some such supposed qualitative item, however, does not in any obvious way involve or require being aware of oneself. Thinking within that framework one thus loses sight of the close relation between phenomenal consciousness and self-awareness. Once one adopts that way of thinking it becomes difficult to see why and to say how phenomenal consciousness and pre-reflective selfawareness are integrated into one another.

Some philosophers might concede that talk about awareness of qualia can be reduced in the way proposed to talk about awareness of experiential properties and still see no problem in continuing using the experience property framework. But this is not, or so I claim, an advisable way to proceed. It is unhelpful to use a framework which hides the important relation between phenomenal consciousness and self-awareness 'under the surface'. It neither contributes to clarity of thought nor to the reliability of intuition to use a language which must first be reduced to a more fundamental and simpler language in order to render theoretically important insights accessible or evident. It is safer to work directly, in such a situation, with the more fundamental and theoretically more transparent terminology.

\section{Higher order theories of consciousness}

According to so-called higher order theories of consciousness to be phenomenally conscious is a matter of being aware of one's own states. A central claim of such theories is thus that there is no necessary link between a state having qualia and a state being phenomenally conscious. This separation makes the view seem bizarre to 
many philosophers who take the problem about phenomenal consciousness seriously. From their perspective to abandon the necessary link between qualia and phenomenal consciousness is to change the subject; it is to use the term "qualia" in a different sense. But the proponent of the higher order theory of consciousness takes the term qualia to be a theoretical term the reference of which is fixed by the role qualia are supposed to play in the explanation of behaviour. Under this presupposition the claim that qualia are conscious has the status of a thesis which cannot be decided by reflection on what qualia are but which requires an argument (supposedly an empirical argument). The friend of necessarily conscious qualia will tend to be puzzled by such a proposal and insist that to suppose that qualia can be phenomenally unconscious is to misunderstand the term. That qualia are phenomenally conscious is not a substantial claim about qualia; it is rather-given the way the term is intended to be understood-trivially true, or so many philosophers would like to reply.

In this context it may be interesting to note that the claim that qualia are necessarily phenomenally conscious comes out as trivially true when the claim is translated in a plausible manner into the framework of experiential properties. On its intended interpretation - at least as it is common among 'friends' of qualia-for a state to have qualia is (expressed within the framework of experiential properties) for the state to be such that the subject has experiential properties by being in that state. This is the sense of "having qualia" that is perfectly acceptable and that philosophers involved in this debate typically have in mind, or so I suggest. But for a state to be phenomenally conscious is - according to common understanding-nothing else. I cannot see any other adequate way to translate the latter locution into the language of experiential properties but the one just proposed for the former locution. Translated into the framework of experiential properties the two assertions "state S has qualia" and "state $S$ is phenomenally conscious" collapse into one and the same claim; they must both be translated as follows: the subject involved in $\mathrm{S}$ has experiential properties by being in state S. "States with qualia need not be phenomenally conscious" then translates into "there are states $S$ that fulfil both of the following conditions: (a) the subject involved in $\mathrm{S}$ has experiential properties by being in $\mathrm{S}$ and (b) the subject involved in $\mathrm{S}$ does not have any experiential property by being in S." This claim is of course trivially false. ${ }^{13}$ If this diagnosis is correct then one may conclude that the prima facie plausibility that many see in higher order theories of consciousness is an artefact of the experience property framework.

\footnotetext{
13 In response the opponent can argue against one of the two translations and/or reformulate the original thesis with respect to experiential properties which would mean to insist that one can have experiential properties without thereby being phenomenally conscious. Experiential properties however are, by stipulation, such that instantiating them just is to be in a state with a specific phenomenology and therefore in a state which is phenomenally conscious. Perhaps the proponent of the higher order theory of consciousness must reply at this point that there is no reason to suppose that any genuine kind of properties can be picked out by that stipulation. I would like to thank David Rosenthal for a conversation about these issues at the APA in San Francisco in April 2016 which helped me a lot to understand the deeper disagreements between the proponent of higher order theories of consciousness and philosophers who take it to be obvious that qualia are phenomenally conscious. This brief section cannot do duty to the dialectic of the situation which became clear in a beautiful manner in that conversation and which I would like to address in a more satisfying manner on a different occasion.
} 


\section{Transparency and attending to phenomenal character}

What has been called 'transparency of experience' has many different interpretations. ${ }^{14}$ But we need not comment on these distinctions here. For the purposes of this section we may focus on one central idea concerning the direction of attention. Several philosophers have pointed out that a subject who tries to attend to the phenomenal character of its own experience will find itself attending to what is phenomenally present in the experience. For instance, when a person tries to focus on what it is like for her to see a tree, then she will focus on the rich variety of colours and shapes of the tree she is looking at. Famously it has been concluded by various philosophers that either there are no qualitative properties of experiences (no qualia) or that those qualia cannot be attended to. ${ }^{15}$

Presupposing that awareness of what it is like to undergo an experience is, as the experience property framework suggests, awareness of qualia in the technical sense here at issue, it follows from the above result that we are not, in undergoing an experience, aware of what it is like to undergo it. This is precisely the conclusion many have drawn: based on the 'transparency of experience' in the sense just explained, it is often argued that there is no reason to assume that experiences have qualitative character or that there is something that it is like to undergo them or that there is no reason to suppose that phenomenal consciousness raises any problem for physicalism.

In the context of this argument the experience property framework plays an unhappy role in two related ways. First, it invites those who think in its terms to conflate two distinct potential results of the argument. Second, and relatedly, it invites a misinterpretation of what the transparency of perception involves. The first mistake consists in the failure to distinguish the following two claims $\mathrm{C} 1$ and $\mathrm{C} 2$ :

$\mathrm{C} 1$ : Experiences do not have qualia or we are never aware of those qualia.

$\mathrm{C} 2$ : It is not true that there is something that it is like to undergo an experience or, if there is something that it is like to undergo an experience, then we are never aware of what it is like to undergo it.

According to the experience property framework $\mathrm{C} 1$ and $\mathrm{C} 2$ tell us exactly the same since within that framework (a) for an experience to have qualia means that there is something that it is like to undergo it and (b) for a subject to be aware of what it is like to undergo an experience is for it to be aware of qualia. But, as argued before, this identification is unacceptable and based on confusion. If the arguments here presented go through, then $\mathrm{C} 1$ is true but $\mathrm{C} 2$ is false.

Here is the second way in which the experience property framework tends to lead one astray when one reflects within it about transparency in the sense sketched above: within a common version of that framework, to be aware of what it is like to undergo an experience is understood as being presented with a quale instantiated by some internal process. If this understanding were adequate, then one should indeed expect that a person who succeeds attending to what it is like to see a tree either turns his or her

\footnotetext{
14 For different interpretations of transparency compare my paper (2007).

15 For well-known versions of the argument see Harman (1990) or Martin (2002) and for critical discussions of this kind of argument compare Kind (2003), Siewert (2004) and my paper (2007).
} 
attention away from the tree in order to direct it 'inside' or simultaneously attends into two opposite directions (to the tree outside there and to the quale instantiated 'inside'). But, obviously, neither the first, nor the second actually happens as a little reflection reveals. We do not turn our attention away from the tree and its perceived properties nor do we simultaneously attend into two different 'directions' when we try to focus on what it is like to see a specific tree on a given occasion. For those who adopt the framework at issue it seems to follow that any such attempt at focussing our attention on what it is like to see a tree fails.

But of course the just mentioned conclusion is unfounded and the contrary impression is a cognitive illusion created by adopting the relevant version of the experience property framework. Once one fully appreciates that to focus on what it is like to undergo an experience is just to focus on what it is like to instantiate the experiential property at issue, the above mentioned expectation reveals itself as clearly mistaken. There is no reason to suppose that to focus ones attention on what it is like to be phenomenally presented with red requires withdrawing attention from the thing one sees as red. To the contrary, to gain a clear and vivid awareness of what it is like to be phenomenally presented with red in perception requires that one focusses attention on how the relevant surface appears which means to focus on the colour the surface outside there appears to have. Doing so one enhances one's awareness of that particular experiential property one presently instantiates compared to other experiential properties one instantiates simultaneously. And this is precisely the thing to do in order to enhance one's awareness of what it is like to have that particular experiential property.

Describing what happens when one focusses attention on what it is like to undergo an experience in this way within the framework of experiential properties also reveals why there is no double direction of attention involved when a subject focusses on what it is like to undergo an experience. Focussing attention on what it is like to undergo a given experience is a matter of voluntarily enhancing the salience of some experiential property (as just described). This does not involve directing attention in a particular direction in any clear sense. The metaphor of direction towards some item in a supposed 'inner' realm is misleading. To enhance the salience of some specific experiential property one presently instantiates is not to focus on something given as happening 'inside' nor is it to focus on something occurring in the stream of consciousness. Therefore, attending to the red outside there and attending to how it is to see the red out there does not involve directing one's attention in two distinct directions.

Abandoning the experience property framework in favour of the framework of experiential properties reveals in the way just sketched that the argument from transparency briefly presented above is based on a false expectation: contrary to what is suggested by the experience property framework, focussing on what it is like to see a certain tree neither requires withdrawing one's attention from the tree nor does it require double direction of attention. Therefore, the transparency of perception in the sense here at issue does nothing to undermine any of the following theses: there is something that it is like to undergo perceptions; we are able to focus on what it is like to undergo a perception; we are aware of what it is like to undergo perceptions while undergoing them. The impression that transparency of perception undermines any of 
these claims is a cognitive illusion created by the experience property framework and its misconception of phenomenal awareness as exhibiting basic intentionality.

\section{Acquaintance by presentation and access to the nature of what one is acquainted with}

There is a difference between acquaintance by presentation and acquaintance by primitive awareness which may be described, metaphorically, by saying something like this: there is 'a distance' between the subject and what it is acquainted with in the case of acquaintance by presentation; there is no such 'distance' in the case of acquaintance by primitive awareness. Intuitively it seems clear that whenever a subject is acquainted with something by presentation, then the object it is thus acquainted with may well have hidden sides which are not revealed to the subject by that kind of acquaintance. Obviously this is so in the subcase of acquaintance by perception or by thought (if there is acquaintance with contents by thinking about them) and it is arguably so in typical cases of acquaintance by phenomenal presentation (e.g. with colours, tastes or sounds). Being presented to someone 'as an object' of experience or thought quite clearly opens up the possibility that 'the object' thus given, in whatever way it might be 'given' to the subject, does not reveal its nature to the subject to whom it is presented.

The situation is different for the case of acquaintance by primitive awareness. Arguably, by being primitively aware of its own experiential properties the subject stands in the closest possible relation to the properties it is thereby acquainted with. There is, in a sense, no 'distance' between the subject and what it is acquainted with in this special case.

Many aspect of the phenomenology of one's own present state go unnoticed. It may be difficult or even impossible to attend to one's own experiential properties in a way which makes the relevant phenomenal aspects of one's present state more salient. Attending to a specific voice in a piece of music and thereby attending to what it is like to hear that voice may unavoidably change the experiential properties one instantiates. Furthermore, there are many cases where it is difficult to tell what having the relevant experiential property consists in. For instance, everybody is acquainted with being under the impression of bringing about something in an active manner. But it is a matter of dispute whether the nature of that experiential property can be accounted for by a description of how it presents the world to be (by its intentional content) and if so how that description would have to look like. So, being acquainted with experiential properties by primitive awareness does not reveal the nature of those properties to the experiencing subject in a way which renders their adequate theoretical description obvious. Nor is it excluded to be in error about the experiential properties one is presently instantiating. Errors may be due to inadequate conceptualization or to a mistake in the formation of the relevant belief. What all this shows is that the subject who is acquainted with experiential properties on the basis of experience thereby does not have direct or infallible epistemic access to those properties. The subject's epistemic access to the experiential properties it is acquainted with by primitive awareness is not immediate. 
But primitive awareness is itself a kind of 'access' to those properties and it is this access which can adequately be said to be direct and immediate and which may be described as involving no 'distance' between the subject and what it is acquainted with. The experiencing subject is aware, in the sense of primitive awareness, with every aspect of the phenomenology of its overall present state. It is aware of every such aspect just in virtue of the fact that such aspects are what they are: aspects of what it is like for the subject to be in its present state. Experiential properties constitute that over-all phenomenology and they are nothing over and above those 'aspects' of phenomenology. Therefore, to be aware in the sense of primitive awareness of instantiating a certain experiential property is to be aware of that property's very nature in a pre-reflective and pre-conceptual way. Acquiring knowledge of that nature by the formation of an adequate concept is a different matter. Primitive awareness of a given experiential property is the basis of the formation of such a concept. If nothing goes wrong-no misconception is involved and no mistake in the belief formation process occurs - then it enables the subject to acquire an adequate and full understanding of what having a certain experiential property consists in.

Adopting the experience property framework distorts our understanding of phenomenal consciousness by misrepresenting the way in which we are aware of what it is like to undergo our own experiences. It portrays awareness of our own experiences as a matter of being presented with something and thereby makes it difficult or impossible to understand the intimate relation in which the subject stands to its own experiential properties. This inadequate picture leads to unreliable intuitions, or so I would like to claim, when one engages in the evaluation of arguments for or against physicalism. Anti-physicalist arguments implicitly or explicitly use the idea that we have, as experiencing beings, a particular access to the nature of experience. As just sketched, that particular access is however miss-described within the experience property framework. ${ }^{16}$

\section{Concluding remark}

The examples given in the preceding sections illustrate that the experience property framework and the framework of experiential properties are not just two variants of one and the same theoretical language. It does matter which of the two frameworks

\footnotetext{
16 Nida-Rümelin (2006) and Goff (2011) both argue for the following claim: to have an experienced-based concept (a so-called phenomenal concept) of an experiential property allows the subject at issue (under ideal circumstances) to grasp (to fully understand) what having the relevant property consists in (this is the thesis called phenomenal essentialism in Nida-Rümelin (2006), phenomenal concepts are, according to this claim nature-revealing, they are transparent in the terminology of Philip Goff). In these two papers some intuitive support is given for phenomenal essentialism but no explanation of how we gain such cognitive access to the nature of these properties is proposed. The present section can be seen as a sketch of such an explanatory account; the account is available only within the framework of experiential properties (it does not seem to have an equally satisfactory counterpart when formulated within the experience property framework). Phenomenal essentialism would of course gain additional support by an account of how a subject can from a nature-revealing concept of an experiential property on the basis of his or her primitive awareness of having the relevant experiential property. An elaborated version of such an explanation must however be left to a different occasion.
} 
one chooses since none of the 4 conditions mentioned earlier in Sect. 2 are satisfied. The examples given show that, to the contrary, the following claims $\left(1^{\prime}\right)-\left(4^{\prime}\right)$ can be justified:

$\left(1^{\prime}\right)$ A claim which has intuitive appeal when formulated in one of the two frameworks is not always as attractive, intuitively, when formulated in the other framework.

This is so, for instance, for the claim that phenomenal consciousness and prereflective self-awareness are necessarily integrated, for the claim that the nature of experiences is revealed in experience, for the claim that states with qualia need not be phenomenally conscious and for claims about the consequences of so-called transparency of perceptual experience. As argued before, intuition goes astray when the experience property framework is used; intuitions that are invited by the framework of experiential properties are more reliable. ${ }^{17}$

$\left(2^{\prime}\right)$ Rational evaluation of an argument when formulated in one of the two frameworks does not always lead to the same result as rational evaluation of the corresponding argument when formulated within the other.

One will come to different results in one's rational evaluation, for instance, with respect to the argument from transparency sketched above and with respect to any antiphysicalist argument based on the idea that experience provides access to the nature of experience. Here again, as has been argued, the experience property framework creates cognitive illusions and undermines reliable judgement.

( $\left.3^{\prime}\right)$ Claims which can be formulated within one of the two frameworks do not always have an adequate translation into the other framework.

For instance, there does not seem to be any satisfying way to translate what has been said about primitive awareness in Sect. 8 into the language of the experience property framework.

$\left(4^{\prime}\right)$ The two frameworks are not equally adequate to the phenomena they conceptualize.

If what has been said in this paper is correct, then the experience property framework invites fundamental misunderstandings with respect to consciousness, selfconsciousness and the relation of the two. To avoid these mistakes the experience property framework should be abandoned in favour of the framework of experiential properties. $^{18}$

Open Access This article is distributed under the terms of the Creative Commons Attribution 4.0 International License (http://creativecommons.org/licenses/by/4.0/), which permits unrestricted use, distribution, and reproduction in any medium, provided you give appropriate credit to the original author(s) and the source, provide a link to the Creative Commons license, and indicate if changes were made.

\footnotetext{
17 This claim is closely related to claim $\left(4^{\prime}\right)$.

18 Chapter 2 of my book "Conscious Individuals. Sketch of a theory", OUP, in preparation, discusses the mistakes that are invited by the experience property framework here described and a few more in greater detail.
} 


\section{References}

Brentano, F. (1874). Psychologie von empirische Standpunkt (Vol. 1, book 2).

Byrne, A. (2004/2015). Inverted qualia. In Stanford encyclopedia of philosophy.

Carpintero, M. G. (2003). Qualia that it is right to Quine. Philosophy and Phenomenological Research, $\operatorname{LXVII}(2), 357-376$.

Chalmers, D. (2002). Consciousness and its place in nature. In D. Chalmers (Ed.), Philosophy of mind. Classical and contemporary readings. New York: Oxford University Press.

Crane, T. (2005/2011). The problem of perception. In Stanford encyclopedia of philosophy.

Gertler, B. (2012). Conscious states as objects of awareness: On Uriah Kriegel, Subjective consciousness: a self-representational theory. Philosophical Studies, 159, 447-455.

Goff, Ph. (2011). A posteriori physicalists get our phenomenal concepts wrong. Australasian Journal of Philosophy, 89(3), 191-209.

Harman, G. (1990). The intrinsic quality of experience. In James Tomberlin (Ed.), Philosophical perspectives 4: Action theory and philosophy of mind (pp. 31-52). Ridgeview: Atascadero.

Horgan, T., \& Kriegel, U. (2007). Phenomenal epistemology: What is consciousness that we may know it so well? Philosophical Issues, 17(1), 123-144.

Jackson, F. (1982). Epiphenomenal qualia. The Philosophical Quarterly, 32(127), 127-136.

Kind, A. (2003). What's so transparent about transparency? Philosophical Studies, 115, 225-244.

Kind, A. (2008). Qualia. International Encyclopedia of Philosophy. A Peer-Reviewed Academic Resource.

Kriegel, U. (2009). Subjective consciousness: A self-representational theory. Oxford: Oxford University Press.

Levine, J. (2006). Conscious awareness and (self)-representation. In Kenneth Williford \& U. Kriegel (Eds.), Self-representational approaches to consciousness. Cambridge: MIT Press.

Martin, M. G. F. (2002). The transparency of experience. Mind and Language, 17(4), 376-425.

Nida-Rümelin, M. (2006). Grasping phenomenal properties. In T. Alter \& S. Walter (Eds.), Phenomenal concepts and phenomenal knowledge: New essays on consciousness and physicalism. Oxford: Oxford University Press.

Nida-Rümelin, M. (2007). Transparency of experience and the perceptual model of phenomenal awareness. Philosophical Perspectives, 21(1), 429-455.

Nida-Rümelin, M. (2011). Phenomenal presence and perceptual awareness: A subjectivist account of perceptual openness to the world. Philosophical Issues, 21(1), 352-383.

Nida-Rümelin, M. (2014). Basic intentionality, primitive awareness, and awareness of oneself. In A. Reboul (Ed.), Mind. Philosophical papers dedicated to Kevin Mulligan: Values and metaphysics. Berlin: Springer.

Nida-Rümelin, M. (2016). Selfawareness, ***.

Nida-Rümelin, M. (in preparation). Conscious Individuals. Sketch of a Theory. Oxford University Press.

Siewert, C. (2004). Is experience transparent? Philosophical Studies, 117, 15-41.

Siewert, Ch. (2012). On the phenomenology of introspection. In D. Smithies \& D. Stoljar (Eds.), Consciousness and introspection. Oxford: OUP.

Soldati, G. (2013). Elements of a phenomenological theory of perception. Rivista di filosofia, 3(2013), $461-484$.

Soldati, G. (forthcoming). Brentano on self-knowledge. In U. Kriegel (Ed.), Routledge handbook of Brentano and the Brentano school.

Tye, M. (2015). Qualia. In E. N. Zalta (Ed.), The stanford encyclopedia of philosophy (Fall 2015 ed.). http:// plato.stanford.edu/archives/fall2015/entries/qualia/.

Zahavi, D. (2014). Self \& other: Exploring subjectivity, empathy, and shame. Oxford: Oxford University Press. 